\title{
Blunt traumatic innominate pseudoaneurysm and left common carotid occlusion with an associated bovine aortic arch
}

\author{
Patrick Wells, MD, and Aaron Estrera, MD, Dallas, Tex
}

W e report a case of innominate artery pseudoaneurysm and left common carotid occlusion with an associated bovine arch managed by bypass grafting followed by innominate and carotid ligation.

\section{Clinical Summary}

A 20-year-old man was seen after a high-speed motor vehicle collision as a restrained driver. Initial vital signs were stable, and he had an intact mental status although he had reportedly lost consciousness at the crash scene. He was noted to have a prominent seat belt mark extending from his right groin to his left shoulder, with depression of the sternum and ribs along this area. Radial pulses were easily palpable and symmetric, and the left carotid pulse was absent. Breath sounds were diminished on the left side. Initial chest radiograph showed a widened mediastinum, left-sided hemothorax, and elevated left hemidiaphragm. A leftsided chest tube was inserted, producing $600 \mathrm{~mL}$ of blood. Computed tomographic scans of the head, chest, abdomen, and pelvis were obtained. Chest computed tomography demonstrated a large mediastinal hematoma, with suggestion of an aortic arch injury and nonfilling of the left common carotid artery with contrast medium (Figure 1, A). An urgent arteriogram showed a proximal innominate pseudoaneurysm, complete occlusion of the proximal left common carotid artery, and an occluded left vertebral artery with associated bovine arch anatomy (Figure 1, B). The patient had no other immediately life-threatening injuries, and he was taken to the operating room for mediastinal vascular repair. A median sternotomy with right supraclavicular extension was performed, and the innominate vein was divided to expose the innominate and proximal right subclavian arteries. The pericardium was entered, and the ascending aorta and aortic arch were exposed, with care taken to avoid entering the proximal innominate pseudoaneurysm. The distal left common carotid artery was exposed through an incision along the anterior border of the left sternocleidomastoid muscle. Anticoagulation with 5000 units of heparin was administered, and bypass grafting was performed with a $14 \times 7$-mm bifurcated graft

\footnotetext{
From the Department of Cardiovascular and Thoracic Surgery, UT Southwestern Dallas, Dallas, Tex.

Received for publication Jan 13, 2005; accepted for publication March 2, 2005.

Address for reprints: Patrick R. Wells, MD, Department of Cardiovascular and Thoracic Surgery, UT Southwestern Dallas, 5323 Harry Hines Blvd, Dallas, TX 75390 (E-mail: patrickrwells@hotmail.com).

J Thorac Cardiovasc Surg 2005;130:928-9

$0022-5223 / \$ 30.00$

Copyright $\odot 2005$ by The American Association for Thoracic Surgery

doi:10.1016/j.jtcvs.2005.03.005
}

from the ascending aorta to the left common carotid artery and then to the proximal right subclavian artery (Figure 2). An internal shunt was used during bypass of the left carotid artery. After successful bypass grafting, a large partial occlusion clamp was used to control the aortic arch at the innominate takeoff. The innominate and left common carotid arteries were controlled with vascular clamps, and then the hematoma was explored. The innominate artery was found to be torn circumferentially at its

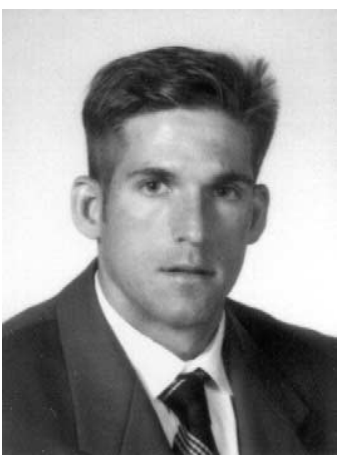

Dr Wells

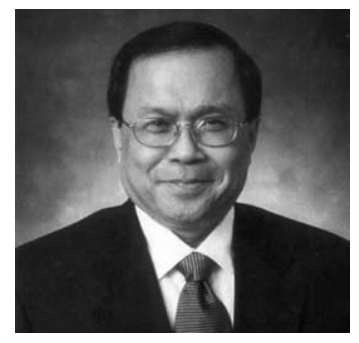

Dr Estrera most proximal portion, and the left common carotid artery was lacerated and thrombosed. The innominate and carotid arteries were divided and ligated. The aortic arch was oversewn with pledget-supported sutures. The patient tolerated the procedure without complication and was neurologically intact on emergence from anesthesia. He had an uneventful postoperative course and was discharged from the hospital 6 days after admission.

\section{Discussion}

Innominate vascular injury is a rare entity, particularly in blunt trauma. It is estimated that $71 \%$ of patients with innominate injuries die before arrival at the hospital. ${ }^{1}$ Bovine arch anatomy is present in $27 \%$ of the population. ${ }^{2}$ Patients with bovine arch anatomy may account for a higher percentage of patients with blunt innominate artery injuries. It is theorized that bovine anatomy predisposes toward injury because there are a decreased number of fixation points on the aortic arch when it is compressed suddenly between the sternum and spine while the neck is hyperextended. The energy of this force is concentrated on the innominate takeoff, resulting in a tear or transection. ${ }^{1}$

Operative repair has been approached in many different ways: interposition or bypass grafting with or without shunts, cardiopulmonary bypass with hypothermic arrest, and combined endovascular and open repairs. ${ }^{1-5}$ There are advocates of each method. but in this particular case, bypass grafting followed by pseudoaneurysm exploration was considered the ideal method. Our patient's entire cerebral perfusion was dependent on the injured but patent innominate artery because of the occluded left common carotid and left vertebral arteries. It was believed that cardiopulmonary bypass with hypothermic arrest was not needed and that the associated risks of systemic heparinization, hemodilution, coagulopathy, and neurologic complications could be avoided. An in- 

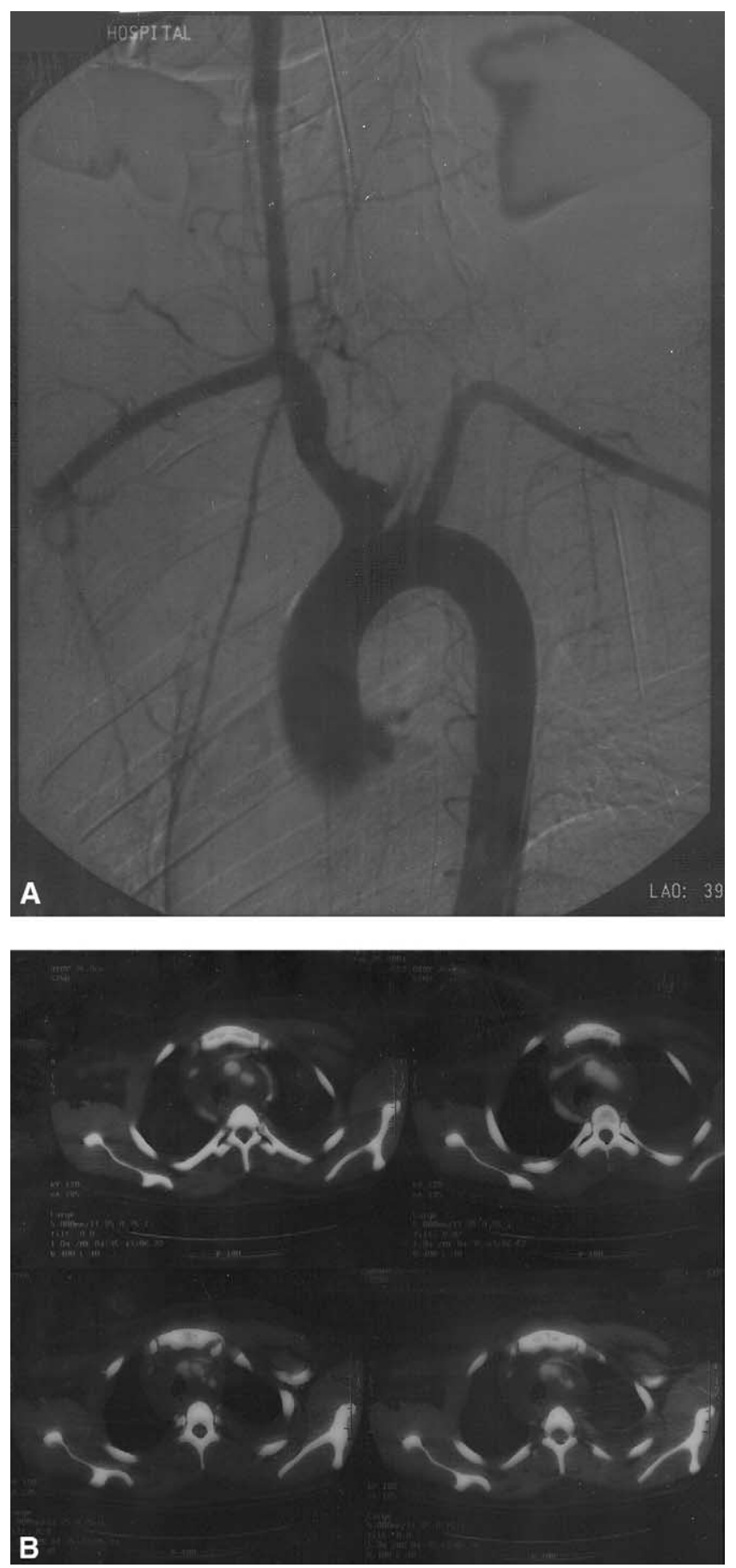

Figure 1. A, Computed tomographic scan of chest. B, Arch aortogram.

ternal shunt was used during bypass grafting to the distal left common carotid artery, and this was placed empirically without measurement of distal carotid stump pressures. We believe that for this injury bypass grafting followed by dissection of the pseudo-

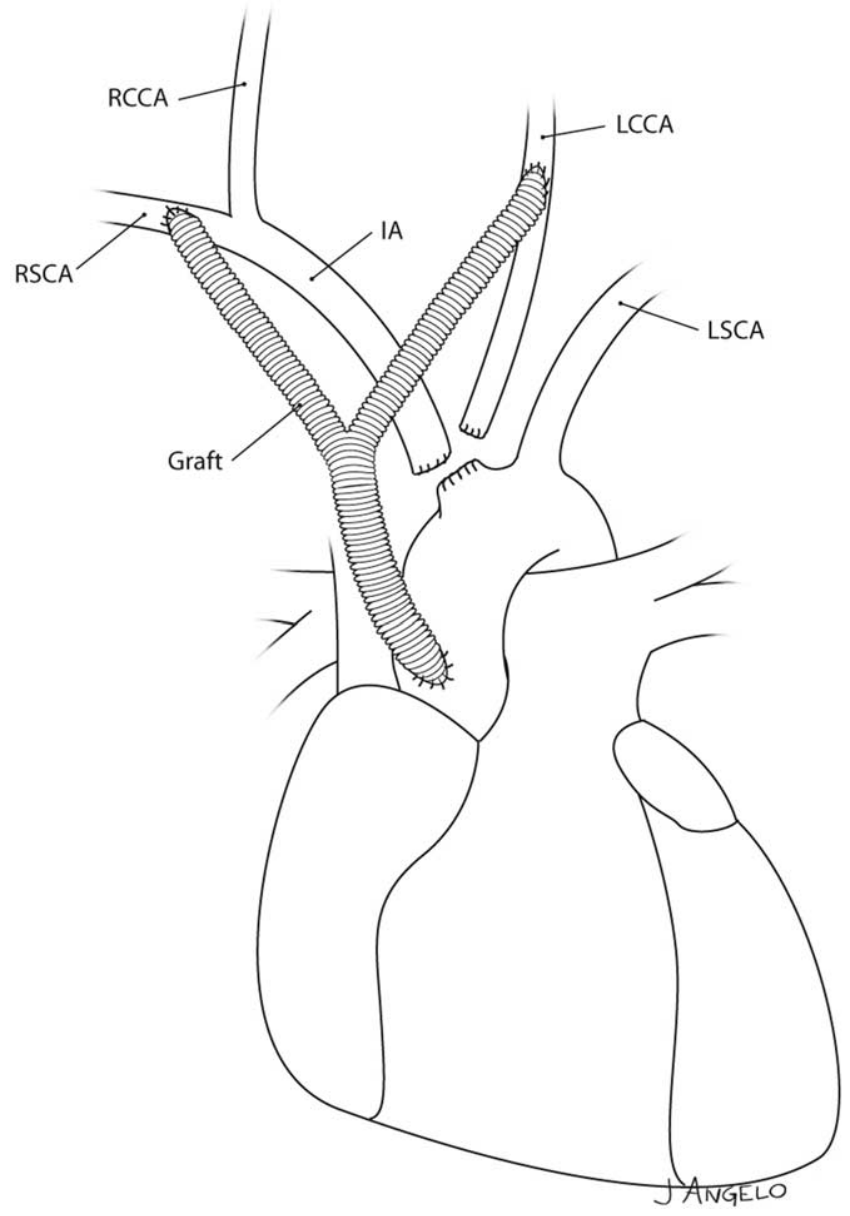

Figure 2. Operative repair diagram. RCCA, Right common carotid artery; LCCA, left common carotid artery; RSCA, right subclavian artery; IA, innominate artery; $L S C A$, left subclavian artery.

aneurysm is the preferred method of repair. Cardiopulmonary bypass with hypothermic arrest is unnecessary unless injury of the aortic arch is extensive or bypass grafting cannot be safely performed before entrance of the pseudoaneurysm.

\section{References}

1. Graham JM, Feliciano DV, Mattox KL, Beall AC. Innominate vascular injury. J Trauma. 1982; 22:647-55.

2. Roberts CS, Sadoff JD, White DR. Innominate arterial rupture distal to anomalous origin of left carotid artery. Ann Thorac Surg. 2000;69: 1263-4.

3. Moise MA, Hsu V, Braslow B, Woo YJ. Innominate artery transection in the setting of bovine arch. $J$ Thorac Cardiovasc Surg. 2004;128: 632-4.

4. Mauney MM, Casada DC, Kaza AK, Merlotti G. Management of innominate artery associated with a bovine arch. $J$ Trauma. 2002;52: $1002-4$.

5. Ruebben A, Merlo M, Verri A, Rossato D, Savio D, Muratore P, et al. Combined surgical and endovascular treatment of traumatic pseudoaneurysm of the brachiocephalic trunk with anatomical anomaly. J Cardiovasc Surg (Torino). 1997;38:173-6. 